FORMATION Formation emploi

Revue française de sciences sociales

113 janvier-mars 2011

La flexicurité à l'aune de l'approche par les capacités

\title{
Post-face : la flexicurité peut-elle encore constituer une ambition pour l'Europe?
}

Dominique Méda

\section{(2) OpenEdition \\ 12 Journals}

Édition électronique

URL : http://journals.openedition.org/formationemploi/3288

DOI : 10.4000/formationemploi.3288

ISSN : 2107-0946

Éditeur

La Documentation française

Édition imprimée

Date de publication : 1 mars 2011

Pagination : 97-109

ISSN : 0759-6340

\section{Référence électronique}

Dominique Méda, «Post-face : la flexicurité peut-elle encore constituer une ambition pour l'Europe? », Formation emploi [En ligne], 113 | janvier-mars 2011, mis en ligne le 10 avril 2013, consulté le 30 octobre 2020. URL : http://journals.openedition.org/formationemploi/3288 ; DOI : https://doi.org/ 10.4000/formationemploi.3288

(c) Tous droits réservés 


\section{DOSSIER}

\section{Post-face : \\ La flexibilité peut-elle encore constituer une ambition pour l'Europe?}

Dominique Méda*

Un peu moins de quatre ans après la publication de la Commission européenne, intitulée «Vers des principes communs de flexicurité : des emplois plus nombreux et de meilleure qualité en combinant flexibilité et sécurité » (juin 2007), ce dossier spécial de Formation Emploi revient de manière non seulement opportune mais aussi extrêmement originale sur les éléments constitutifs de ce mot-valise, sur les diverses interprétations auxquelles il a donné lieu et, surtout, sur le bilan susceptible d'être tiré des actions qui ont été mises en œuvre en son nom. S'inscrivant dans une double ambition théorique et pratique et dans un cadre d'analyse commun - celui de la théorie des capacités développé par Amartya Sen -, l'ensemble des cinq articles qui précèdent constitue en effet un matériau précieux pour une évaluation raisonnée des effets des politiques susceptibles d'être rangées sous la bannière de la flexicurité.

L'un des grands intérêts de ce numéro est très certainement d'abord de nous obliger à réfléchir sur les cadres, les variables et les critères à mobiliser pour évaluer ce que la Commission européenne considère comme une " stratégie » : " la flexicurité peut être définie comme une stratégie intégrée visant à améliorer simultanément la flexibilité et la sécurité sur le marché du travail $»^{1}$. Mais comment évaluer une stratégie, à l'aune de quels critères, à l'aide de quels indicateurs, par rapport à quels objectifs ? Le dossier propose en réalité, de manière implicite, une réflexion approfondie sur ce que signifie évaluer. Évaluer, cela peut consister - sans interroger les postulats sur lesquels repose ladite stratégie, ni la configuration, les objectifs et le périmètre qu'elle propose - à regarder si et dans quelle mesure les objectifs affichés ont été atteints et finalement à comparer, de manière statique et dynamique, les évolutions manifestées par des indicateurs dûment choisis. Plusieurs des articles rappellent ainsi, à juste titre, dans la lignée des travaux fondateurs de Salais (2004), combien le taux d'emploi a constitué un indicateur particulièrement central dans la mise

\footnotetext{
${ }^{1}$ Commission européenne, «Vers des principes communs de flexicurité » (COM (2007)359 final), 27 juin 2007.
}

* Dominique Méda est sociologue, directrice de recherches au Centre d'études de l'emploi. Dernier ouvrage: L'Emploi en ruptures, sous la direction de B. Gomel, D. Méda, E. Serverin, Dalloz, 2009 Travail. La révolution nécessaire, Les éditions de l'Aube, 2010. 
en œuvre de la Stratégie européenne de l'emploi et dans son évaluation. Le taux d'emploi, le taux de retour à l'emploi, l'accès à la formation dans les quatre dernières semaines et toute une série d'autres indicateurs constituent aujourd'hui encore les outils principaux permettant le plus souvent de juger de la réussite ou non d'une action. Mais l'ensemble de ces articles invite à interroger non seulement la pertinence des indicateurs sélectionnés pour rendre compte des actions, mais plus généralement celle des politiques mises en œuvre vis-à-vis de l'objectif affiché consistant à améliorer la situation des personnes, et finalement celle de la stratégie elle-même...

Le présent article revient, en premier lieu, sur le bilan mitigé que tirent les auteurs de la mise en œuvre de la stratégie de flexicurité (partie 1), avant de s'intéresser aux raisons alléguées pour expliquer celui-ci (partie 2). Il s'attarde ensuite sur les conditions de réussite d'une politique qui aurait pour objectif moins la mise en place et la consommation de «mesures » que le développement d'un travail de qualité (partie 3), avant de se demander s'il faut pour cela développer une alternative à la conception actuelle de la flexicurité ou plutôt à la flexicurité elle-même (partie 4).

\section{QUEL BILAN TIRER DE LA STRATÉGIE DE FLEXICURITÉ ?}

Revenons donc sur les différentes étapes de ce bilan de la flexicurité que nous permet de dresser ce numéro. Ce n'est pas à un bilan général que nous convient les auteurs, mais plutôt à l'évaluation d'une partie de cette stratégie, et même plus précisément de l'une des composantes de celle-ci. Mais d'une composante majeure. Dans sa communication, la Commission a en effet indiqué que les politiques de flexicurité pouvaient être conçues et mises en œuvre à travers quatre composantes politiques : la souplesse et la sécurisation des dispositions contractuelles; les stratégies globales d'apprentissage tout au long de la vie; des politiques actives du marché du travail efficaces ; des systèmes de sécurité sociale moderne. Cette stratégie peut être présentée brièvement de la manière suivante : la protection de l'emploi (job) étant devenue nuisible à l'emploi, il importe de lui substituer une protection des transitions d'un emploi à un autre (employment), grâce à la souplesse des dispositions contractuelles, et donc de permettre aux personnes de passer très rapidement d'un emploi à un autre. Cela exige, outre des systèmes de sécurité sociale modernes (qui incitent au retour à l'emploi), un entretien par les individus de leur capital humain et de leur employabilité qui devient la source principale de leur possible réaffectation dans un emploi. La formation tout au long de la vie (FTLV) devient donc une composante majeure de la stratégie de flexicurité

La question à laquelle les cinq auteurs contribuent à répondre est donc celle-ci : la formation tout au long de la vie a-t-elle rempli les attentes qui pesaient sur elle ? Est-elle parvenue à sécuriser les parcours professionnels, alors même que ceux-ci sont de plus en plus interrompus ? Est-elle parvenue et peut-elle parvenir à jouer ce rôle central qui lui est désormais dévolu, dès lors que la sécurité n'est plus fondée sur l'occupation de longue durée d'un emploi mais au contraire sur la capacité et la nécessité de changer d'emploi de plus en plus souvent qui caractérisent - ou qui devraient désormais caractériser, selon la Commission européenne - des marchés du travail modernes. Autrement dit encore, la FTLV, censée aider les salariés à entretenir leurs compétences et leur «employabilité » de manière à leur permettre de retrouver un emploi le plus vite possible dès lors que celui qu'ils occupaient aura disparu (ou qu'ils auront dû quitter celui-ci), estelle parvenue à garantir un genre de sécurité équivalent à celui que procurait l'occupation de longue durée d'un emploi ?

À la question formulée pour répondre à une évaluation de premier degré - la formation dispensée dans le cadre des actions entreprises ces dernières années a-t-elle réussi à améliorer la sécurisation des parcours professionnels ? -, les auteurs répondent tous, en utilisant des indicateurs simples, par la négative. La formation, principale composante de la flexicurité, ne permet pas de compenser, ni a fortiori de prévenir l'insécurisation des parcours professionnels, qui a d'ailleurs considérablement progressé. Qu'il s'agisse de la France, de la Belgique, de la Catalogne ou de l'Argentine, les auteurs mettent en évidence non seulement la très grande transformation des parcours (avec beaucoup d'allers et retours entre emploi et chômage et une très forte augmenta- 
tion de la précarité, notamment en Catalogne), mais également une incapacité des actions de formation, telles qu'elles ont été développées, à contrebalancer ou compenser cette augmentation de l'insécurité des parcours et à l'anticiper, comme le voudrait pourtant une représentation mythique de la flexicurité. Cela ne signifie évidemment pas que la formation n'est pas susceptible, dans certaines conditions, de corriger ou d'éviter l'insécurité. Mais les actions de formation, telles qu'elles ont été engagées, par exemple dans les politiques wallonnes de lutte contre le chômage, en Argentine, en Catalogne ou en France, mettent toutes en évidence les défauts classiques et soulignés depuis bien longtemps par les chercheurs : la formation va aux plus diplômés (avec un seul contre-exemple dans les politiques wallonnes qui semblent avoir réussi à donner un surcroît de formation aux femmes très en difficulté) ; les actions de formation sont trop courtes, très utilitaristes et vont rarement au-delà d'une tentative d'adaptation des salariés aux postes de travail existants ou aux desiderata des entreprises. Certes, un tel constat était de mise bien avant l'invention du concept de flexicurité ; cependant, les articles du présent numéro ont le mérite de révéler que, malgré l'importance centrale accordée à la formation dans la stratégie de flexicurité, les principaux défauts des politiques de formation n'ont pas été corrigés, rendant dès lors cette stratégie elle-même peu efficace.

Mais les auteurs n'en restent pas à ce premier niveau. L'analyse et la démarche d'évaluation parviennent à une seconde étape lorsque les auteurs s'accordent sur le fait que les politiques actuelles de formation, élément central de la flexicurité, sont défaillantes parce qu'elles sont considérées comme des actions standardisées, toutes conçues sur le même patron; alors qu'elles devraient pouvoir être adaptées aux situations spécifiques (des territoires, des contextes historiques, des entreprises, et bien sûr des individus) pour pouvoir «fonctionner» correctement. C'est à partir de ce moment que la mobilisation de la théorie des capacités de Sen commence à produire son plein effet : chacun des auteurs met en évidence, à sa manière et sur son terrain, comment l'oubli des conditions qui peuvent permettre l'actualisation d'une potentialité (ou dans le langage de Sen la conversion des ressources en véritables libertés) peut rendre complètement inefficace une politique. Dans le cas de la formation, et sur l'exemple espagnol, Verd met parfaitement en évidence comment, pour produire l'effet attendu, les dispositifs de formation existants devraient, d'une part, être adaptés à la situation historique qui se caractérise par une explosion de la précarité et, d'autre part, comment des politiques publiques adéquates devraient être développées pour permettre aux individus de s'emparer réellement des mesures existantes : «ce sont les circonstances d'utilisation et non l'utilisation en elle-même qui rendent la formation efficace ». Cela signifie que loin d'en avoir fini avec l'action lorsque des ressources, ou des mesures, ont été «mises en place» ou « ouvertes», les pouvoirs publics ou les entreprises ont encore à développer l'ingénierie qui permettra aux personnes de se saisir réellement de ces mesures. Et peut-être cette seconde dimension est-elle encore plus importante que la première. Cette ingénierie doit de surcroît être adaptée aux circonstances particulières et aux individus euxmêmes, puisque « des moyens semblables produisent des effets différents (capabilités différentes) en fonction de facteurs (structure du marché du travail, politiques d'embauche des entreprises...) difficilement contrôlables par les individus ». Il y a là une leçon particulièrement importante pour les concepteurs et les promoteurs des politiques publiques.

La formation, telle qu'elle est actuellement conçue et pratiquée dans les pays analysés est donc un échec, du simple point de vue du maintien dans l'emploi ou du retour à l'emploi, ou encore de la sécurisation des parcours professionnels. Ce constat s'appuie sur un premier bilan réalisé avec les indicateurs et objectifs traditionnels. Mais c'est aussi un échec, poursuivent les auteurs, si l'on reconnaît que la formation ne doit pas seulement servir à adapter la main-d'œuvre aux postes de travail ou aux demandes des entreprises, à augmenter l'employabilité des salariés et à servir au retour rapide à l'emploi dans une optique radicalement utilitariste, et qu'en utilisant la grille d'analyse de Sen, on considère que la formation devrait également (ou avant tout?) constituer un instrument au service du développement personnel, ou au moins permettre aux individus d'exercer une certaine liberté de choix entre différentes options. Or, Verd et LópezAndreu montrent que les accomplissements professionnels des travailleurs et travailleuses sont peu influencés par le recours à des dispositifs de formation 
et que ces derniers n'améliorent pas l'ensemble des options en matière d'emploi auxquels ils ont accès. De la même manière, utilisant les critères de Sen (de quelles libertés de choix les individus ont-ils pu disposer?) pour évaluer l'efficacité des politiques wallonnes, Conter et Orianne montrent qu'une partie des chômeurs ayant suivi une formation l'a fait pour éviter des sanctions administratives ou pour répondre à une demande de leur conseiller emploi. D'autres ont dû accepter l'emploi proposé une fois la formation terminée. La mobilisation des analyses de Sen permet ainsi de mettre en évidence, d'une part, qu'au-delà du seul point du retour à l'emploi, la formation n'a pas pleinement joué son rôle ; d'autre part, que le développement de l'employabilité individuelle n'a que peu d'effets sur les capacités des chômeurs. C'est donc à un constat relatif d'échec que parviennent les auteurs. Mais le grand intérêt de leurs analyses a trait à l'explication rigoureuse et systématique qu'ils tentent de donner de celui-ci.

\section{POURQUOI UN BILAN AUSSI MITIGÉ ?}

Plusieurs explications sont en effet mobilisées pour comprendre ce bilan mitigé de la composante centrale de la flexicurité, et, plus largement, de celle-ci. La première, rappellent Caillaud et Zimmermann, réside dans l'interprétation extrêmement restrictive que la France a faite de la notion de Lifelong learning. Notre pays n'a rien fait d'autre que de plaquer ses pratiques antérieures sur ce concept aux origines très différentes, tout en oubliant d'ailleurs lui-même toute une partie de son héritage. En effet, comme le rappellent Conter et Orianne, citant Dubar, la formation continue a au moins trois fonctions : «une fonction économique d'adaptation de la main-d'œuvre aux changements technologiques ou organisationnels du travail, une fonction sociale d'accès aux différents niveaux de qualification et une fonction culturelle ou idéologique censée favoriser un plus grand épanouissement personnel et une plus grande participation sociale des intéressés ». Caillaud et Zimmermann opposent à la conception large qui est celle de la Charte des droits sociaux, - avec un accent particulier mis sur la personne et ses droits -, celle finalement adoptée par la loi française, après le rejet de l'idée de formation qualifiante différée : « restreinte à un contenu uniquement professionnel, la formation continue à la française ne constitue pas un droit réel de formation tout au long de la vie pour les salariés. Elle s'entend à titre principal comme formation d'adaptation professionnelle. » L'ensemble des articles le mettent en évidence à l'envi : la formation professionnelle, telle qu'elle a été inscrite dans la loi française et mise en œuvre, constitue une considérable réduction tant vis-à-vis de la notion de Lifelong learning que des ambitions dont la loi française de 1971 était porteuse.

Les mêmes évolutions sont constatées en Catalogne, en Belgique et en Argentine ; à cet égard, les auteurs soulignent le caractère étroitement utilitaire de la formation, mise au service d'une « logique de compétences » et d'un retour rapide à l'emploi, qui rend de surcroît le salarié seul responsable du maintien de son employabilité. Plus précisément, malgré le caractère plus large de la notion de Lifelong learning, la formation telle qu'elle est envisagée dans la stratégie de flexicurité par la Commission européenne, apparaît elle aussi bien étriquée : tout se passe comme si la stratégie de flexicurité avait elle aussi déterminé un usage particulièrement restrictif et utilitariste de la formation: "la formation telle qu'envisagée dans le discours sur la flexicurité est donc une forme adaptative centrée sur les aptitudes à l'emploi et sur les compétences utiles au poste de travail.» (Conter, Orianne)

Mais certains des auteurs du dossier avancent une autre hypothèse pour expliquer pourquoi la formation est principalement devenue un instrument d'adaptation des salariés au poste de travail et non un outil de développement professionnel. Il faut, pour le comprendre, revenir à la conception même de la notion de flexicurité, à la philosophie qui a inspiré son invention et plus précisément encore à la notion de sécurité à laquelle celle-ci fait référence. Mobilisant les indicateurs utilisés pour rendre compte des évolutions des États membres en matière de sécurité dans l'emploi comme des révélateurs des postulats sous-jacents aux choix des concepteurs, Bonvin, Moachon et Vero soulignent combien la volonté d'augmenter les taux d'emploi est au cœur de la stratégie européenne, de même que l'accès à l'emploi, quelle que soit la qualité de celui- 
ci ou les circonstances de son obtention. Ils mettent dès lors en évidence la conception de la sécurité sousjacente à la stratégie de flexicurité de la Commission européenne : "la conception de la sécurité qui s'affiche à la lumière de l'indicateur dynamique envisage la sécurité sous l'angle de l'adaptation aux exigences du marché du travail, même si cela implique, pour y arriver, d'une part, de sacrifier la qualité de vie ou de travail, ou, d'autre part, de renforcer la précarisation de l'emploi sur le marché du travail. »

Si la formation est conçue dans sa dimension la plus utilitariste, c'est parce que la conception de la sécurité à laquelle se réfère la Commission est également extrêmement restrictive ; la sécurité étant considérée comme issue de transitions réussies du chômage ou de l'emploi à l'emploi, sans prise en considération de la qualité de celui-ci ni des conditions concrètes d'accès. C'est également pour cette raison que les instruments d'évaluation de la flexicurité ne font pas de place - ou une place restreinte - à la question de la qualité de l'emploi obtenu et plus généralement à la qualité du processus d'obtention de l'emploi (l'existence de choix et la possibilité d'avoir exercé celuici). Utiliser, pour évaluer la pertinence de l'actuelle stratégie de flexicurité, les indicateurs mis en place par la Commission, c'est accepter la conception de la sécurité de l'emploi et de la sécurisation des parcours qu'elle prône, et y rester enfermé.

Cette forte critique de Bonvin, Moachon et Vero rejoint celle d'autres auteurs, notamment de Pascale Vielle $(2007)^{2}$, qui avaient souligné très tôt le caractère extrêmement réducteur de la notion de sécurité à laquelle la Commission se référait. Ils en appelaient à une conception étendue, seule susceptible de répondre aux difficultés concrètes et aux obstacles auxquels les salariés sont confrontés pour engager une mobilité ou revenir sur le marché du travail. Le document de la Commission " $n$ 'élabore pas un cadre renouvelé et mobilisateur de la sécurité sociale, susceptible d'intégrer les nouveaux risques et éventualités qu'affrontent les citoyens européens dans leurs trajectoires professionnelles et familiales » écrivait ainsi Pascale Vielle en octobre 2007.

Dès lors, comme cette dernière, les auteurs en appellent à une autre conception de la flexicurité, une

${ }^{2}$ mais aussi Tangian, 2006 ou Keune et Jepsen, 2007. conception «alternative » dans laquelle la sécurité serait pensée autrement et où la formation pourrait continuer à constituer le cœur de la stratégie mais sous la figure radicalement différente du développement professionnel. Ce qui me semble particulièrement important dans cette tentative de penser une conception alternative de la flexicurité, c'est le raisonnement mobilisé par les auteurs pour montrer la nécessité d'une telle démarche. Il ne s'agit pas de changer de conception parce qu'une autre serait meilleure. Non, une autre conception de la flexicurité est nécessaire pour atteindre les objectifs affichés par la Commission, c'est-à-dire pour rendre simplement possible la sécurisation des parcours. Comme l'indiquent Bonvin, Moachon et Vero dans leur conclusion : « la question est de comprendre si les conditions sont effectivement réunies pour que les salariés disposent d'une liberté réelle de travailler et de se développer professionnellement, ce qui constitue une condition préalable $\underline{3}$ pour prendre une part active dans la transition externe d'un emploi à l'autre ou dans la transition interne à une entreprise visant un changement de poste, de service ou de métier. »

Il s'agit donc essentiellement de mettre en œuvre les conditions nécessaires à l'effectivité, au devenir effectif des mesures de formation mises en place, qui apparaissent comme autant de ressources dont il faut encore organiser l'appropriation par des bénéficiaires potentiels. Dès lors, les très beaux passages que Caillaud et Zimmermann consacrent au développement professionnel, et notamment à la manière dont l'entreprise Bigtrucks organise continûment un espace de délibération et d'expression des choix des salariés, doivent être compris non pas comme la description d'un idéal inaccessible ou d'une voie radicalement autre que celle recherchée par la flexicurité mais au contraire comme la clé de l'effectivité de celle-ci. Les organisations capacitantes y apparaissent comme les plus aptes, finalement, à assurer la réalisation de l'objectif de sécurisation des parcours professionnels parce qu'elles sont les seules à avoir vraiment fourni aux individus les moyens de se saisir des dispositifs existants. Dans l'exemple concret présenté, le rôle de l'organisation consiste non seulement à informer sur les mesures qui existent en matière de formation

\footnotetext{
3 Souligné par Dominique Méda.
} 
mais surtout à fournir les aides nécessaires permettant concrètement aux salariés de s'engager dans les démarches exigées (on sait combien cette aide est importante aussi par exemple pour monter les dossiers de VAE - validation des acquis de l'expérience), et aussi à permettre aux salariés d'être des acteurs de leur développement professionnel en organisant leur participation à l'ensemble du processus de travail.

\section{L'ORGANISATION « CAPACITANTE » ET LA QUALITÉ DE L’EMPLOI}

Arrêtons-nous un instant sur cette organisation « capacitante », que Caillaud et Zimmermann décrivent comme celle qui permet aux salariés d'apprendre en situation de travail : elle est très proche de ce que la littérature décrit sous le terme d' " organisation apprenante ». Exploitant les enquêtes Conditions de travail de la Fondation de Dublin, Valeyre et Lorenz (2005) ont ainsi mis en évidence la diversité des types d'organisation du travail en Europe et le fait qu'à côté des organisations en «lean production » («production allégée ») se sont également développées, depuis une trentaine d'années, des « organisations apprenantes ». Celles-ci se caractérisent par une plus grande autonomie et initiative des salariés dans le travail et des pressions temporelles moindres, ce qui leur permet de mieux réguler leur travail et de bénéficier, au final, de meilleures conditions de travail que les autres salariés. Deux éléments importent dans ce rapprochement : d'une part, la répartition des types d'organisation obéit à des logiques géographiques (les organisations apprenantes sont plus répandues dans les pays du Nord). D'autre part, cette répartition présente de forts recoupements avec la «cartographie » de la qualité de l'emploi (Davoine, Erhel, 2007) qui comporte elle aussi une forte diversité.

Il n'est pas indifférent que Caillaud et Zimmermann, cherchant à préciser les contours et les caractéristiques de l'organisation capacitante, insistent sur le fait que les accords collectifs et les politiques des ressources humaines ne suffisent pas et que le management et l'organisation du travail sont décisifs, mais aussi qu'ils mettent au cœur du développement professionnel la qualité du travail et de l'emploi. Si le travail de qualité ne dépend pas du seul travailleur ni de la seule entreprise mais résulte d'une co-construction, alors l'organisation du travail dans son sens le plus large est essentielle; de même, la manière dont l'entreprise suscite la participation et l'expression des salariés, leur permet ou non d'exercer leurs choix et de « donner de la voix », est déterminante, non seulement pour la qualité du travail fourni mais également pour la qualité du travailleur lui-même. Comme l'indiquent les auteurs, c'est bien parce que ce processus d'apprentissage, et même plus largement de développement, est de qualité, que les salariés issus de ce genre d'entreprise sont reconnus et bénéficient euxmêmes d'une présomption de qualité. Notons enfin la proximité entre ces résultats de recherche, qui mettent en évidence la meilleure capacité de ces organisations capacitantes à produire de la sécurité, et ceux d'autres travaux, comme ceux d'Isabelle Ferreras (2007) qui s'intéresse aux salariés travaillant dans des organisations plutôt tayloristes et qui revendiquent leur droit à être traités comme des citoyens ou au moins sur un registre qui n'est pas celui de l'ordre domestique mais de l'ordre politique.

Dès lors, ce dossier permet de revenir, pour le déplorer, sur un double oubli : au niveau le plus macro, oubli par la Commission européenne de la qualité de l'emploi qu'elle avait prônée à une époque et mise à l'agenda, un oubli organisé au nom de la priorité à donner à la quantité d'emplois. Au niveau micro, oubli des conditions élémentaires de production d'un travail de qualité, mais également des conditions d'effectivité des mesures censées permettre le développement de la stratégie de flexicurité telle que l'imaginait la Commission. Oubli, enfin, des critères d'évaluation alternatifs, susceptibles de révéler non pas des augmentations purement faciales de la consommation de mesures qui ne débouchent sur aucune sécurité réelle, mais des améliorations du bien-être au travail, de la maîtrise des salariés sur leur destin, de la qualité du travail - dans son double sens de produit et de processus de production. La correction de cet oubli constituerait d'ailleurs un vrai défi : l'amélioration du développement professionnel peut-elle être mesurée ? À l'aide de quels indicateurs ? Cette question nous rappelle combien l'adéquation d'un indicateur à son objet est complexe et combien l'opération de mesure 
elle-même, de quantification, emporte toujours le risque de focaliser l'attention sur la partie de la réalité éclairée par l'indicateur aux dépends du reste (Desrosières, 2008).

\section{UNE ALTERNATIVE À LA CONCEPTION ACTUELLE DE LA FLEXICURITÉ OU UNE ALTERNATIVE À LA FLEXICURITÉ ?}

Comme beaucoup d'auteurs (Elbaum, 2004, Vielle, 2007, Keune et Jepsen, 2007, Méda, 2009; Bonvin, Vieille, 2009; Keune, Pochet, 2009), les auteurs rassemblés dans ce numéro, et particulièrement Bonvin, Moachon et Vero, proposent " une autre conception de la flexicurité et donc d'autres instruments et indicateurs pour la mesurer $»$. À la recherche des conditions nécessaires pour obtenir les transitions " réussies » auxquelles aspire la Commission, la plupart des auteurs cités parviennent à la conclusion que ce qui importe est de permettre aux personnes de prendre des risques. Il faut pour cela leur permettre de bénéficier à la fois d'un socle de mesures, de l'appui actif d'institutions bienveillantes, de services publics réactifs, de politiques et d'institutions précisément organisées pour donner des étais aux individus et les aider à convertir des ressources en libertés réelles d'agir. La plupart reconnaissent de ce fait que la conception de la sécurité à laquelle la Commission se réfère est grandement insuffisante. Ce simple fait pourrait suffire à invalider l'usage du terme de flexicurité. Mais d'autres éléments vont dans ce sens. On revient ci-après sur l'histoire de la notion de flexicurité, et sur le fait qu'elle apparaît bien comme l'habillage nouveau de revendications anciennes, avant de présenter une série d'arguments qui favorisent l'abandon de la stratégie de flexicurité plutôt qu'une improbable transformation interne.

Revenons donc aux origines et rappelons les raisons pour lesquelles le concept ou le mot-valise de flexicurité a pu être adopté, alors que les tentatives précédentes d'augmenter le recours à la flexibilité s'étaient heurtées, dans un certain nombre de pays, à de fortes résistances. Wilthagen et Tros (2004), Tagian (2006), Keune et Jepsen (2007) ont rappelé les origines danoises et néerlandaises du concept : il est très important de reconnaître combien le fait que le Danemark et les Pays-Bas - pays encore considérés comme appartenant au modèle social-démocrate à l'époque - ont été érigés en modèles, a compté dans l'adoption de la notion de flexicurité (ou au moins dans la moindre résistance opposée originellement à son introduction dans certains pays), comme l'a bien relevé Tagian (2006). En mettant en avant ces deux pays, on insistait notamment sur le niveau élevé des dépenses pour le marché du travail, sur le caractère « généreux » de l'indemnisation du chômage, sur l'existence d'institutions susceptibles d'accompagner activement les demandeurs d'emploi; de même, on attirait l'attention sur les caractéristiques du modèle nordique : niveaux élevés des dépenses de protection sociale, fort développement de l'État-Providence, développement précoce (dès les années 60 ) des politiques actives d'emploi. Sans doute le fait que des pays ancrés dans une telle tradition aient de surcroît réussi à réduire considérablement leurs taux de chômage après la crise des années 90 , et à exhiber un haut niveau de fluidité sur le marché du travail, a vaincu les dernières résistances.

Mais la considération attentive des années antérieures permet de comprendre que le recours au terme de flexicurité - dont la plupart des auteurs ont pointé le caractère de mot-valise commode et d'oxymore (Tagian, 2006 ; Freyssinet, 2008 ; Serrano, 2007) - consiste à revêtir de vieilles idées d'habits neufs. Notamment, l'analyse des textes consacrés régulièrement aux causes des dysfonctionnements des marchés du travail européens par l'OCDE, depuis la fin des années 80 (OCDE, 1990 ; OCDE, 1994), révèle que le terme de flexicurité rassemble, sous un seul vocable, les deux objectifs assignés par l'organisation aux pays européens depuis l'appel à devenir une " société active» : augmenter la flexibilité des marchés du travail - principalement par le biais d'une réforme structurelle du marché du travail passant par l'affaiblissement de la protection de l'emploi - et élever les niveaux de qualification de la population. Les deux objectifs correspondent à la représentation du fonctionnement idéal du marché du travail sous la figure de la « destruction créatrice » chère à Schumpeter : il importe de laisser disparaitre les emplois non rentables et de permettre aux salariés de retrouver au plus vite un autre emploi ; 
et ce, en instaurant les incitations nécessaires pour qu'ils ne s'installent pas dans le chômage ou l'inactivité, et en comptant sur la formation pour leur permettre d'entretenir leur employabilité. Rappelons deux des neuf recommandations qui concluent l'étude de l'OCDE sur l'emploi 1994 : "revoir les dispositions relatives à la sécurité de l'emploi qui freinent son expansion dans le secteur privé» (recommandation $\mathrm{n}^{\circ} 5$ ) et «améliorer les qualifications et les compétences de la main-d'œuvre en modifiant profondément les systèmes d'enseignement et de formation » (recommandation $\mathrm{n}^{\circ} 9$ ). L'insistance de l'OCDE à diffuser ces recommandations et le grand nombre de travaux consacrés à la nécessaire réforme structurelle du marché du travail (pourtant mise en doute par Malinvaud (2003) lui-même), s'est appuyée sur nombre de mises en évidence de corrélations entre la rigueur de la législation protectrice de l'emploi et le chômage. Le fait que l'année précédant la publication de la Commission sur la flexicurité ait été celle de la publication du livre vert «Moderniser le droit $d u$ travail» (2006), dont l'introduction rappelle que : « La modernisation du droit du travail est l'une des principales conditions d'une capacité d'adaptation des travailleurs et des entreprises qui produit des résultats », n'est évidemment pas sans importance.

Il n'est pas possible de développer ici plus avant l'hypothèse d'un « habillage » plus seyant d'une idée ancienne. Il suffit de retenir, comme beaucoup d'auteurs en conviennent, que parmi les quatre composantes de la flexicurité, la souplesse des dispositions contractuelles tient une place très importante - la première au moins dans l'ordre d'apparition. De même, le principal objectif recherché par les promoteurs de la flexicurité était bien d'engager une réforme structurelle du marché du travail pour substituer à la protection de l'emploi la protection des personnes ; ainsi, la notion de sécurité, auparavant ancrée dans l'emploi (au sens du poste de travail et du contrat liant un employeur et un salarié) changeait radicalement de sens puisqu'elle s'entendait désormais comme le passage, la transition entre deux emplois. Il y a là un premier argument qui permet de mesurer la difficulté à accommoder le concept de flexicurité différemment. Est-il possible de remplir différemment ce concept certes très malléable et dont l'acclimatation a réussi en partie du fait de sa plasticité et de la multiplicité des interprétations possibles dont il pouvait faire l'objet avec un tel point de départ? Il n'existe en effet que deux options : changer radicalement la notion de sécurité dans laquelle s'enracine le terme et lister les sécurités de base auxquelles les salariés européens doivent pouvoir accéder, ou changer de stratégie de référence. Une conception alternative de la sécurité dans le cadre de la flexicurité ou... un abandon de la stratégie de flexicurité.

Ajoutons à ce premier argument (la notion de sécurité dans laquelle s'ancre la flexicurité est gravement insuffisante) un autre, également dirimant et qui concerne les usages qui ont été faits du terme : son utilisation a recouvert plus de mesures visant à augmenter la flexibilité qu'à augmenter les sécurités. En France, le terme a été vraiment adopté par le pouvoir politique pendant les années 2004 et 2005. Après un usage encore prudent du terme par le ministre du Travail, de l'Emploi et de la Cohésion sociale, au moment de la mise en place du plan de cohésion sociale, et par le sénateur Pierre Méhaignerie, auteur d'un rapport sur le modèle danois, le terme est revendiqué par Dominique de Villepin, à l'occasion de la mise en œuvre du Contrat Nouvelle Embauche (CNE) ; ce nouveau type de contrat permettait à l'employeur de ne pas motiver le licenciement de son salarié pendant deux années et était censé concilier souplesse pour l'employeur et surcroît de sécurité pour le salarié. Comme nous l'avons montré au terme d'une recherche sur des salariés embauchés en CNE, ce contrat a constitué, en même temps que la première véritable occurrence publique du terme, le premier échec du développement d'une flexicurité à la française (Gomel, Méda, Dalmasso, Schmidt, 2007). En effet, alors même que le donnant-donnant ne concernait que l'équilibre du nouveau contrat, une prime de rupture et un accompagnement renforcé étant échangés contre la possibilité d'être licencié sans motifs, nous avons montré que non seulement l'accompagnement renforcé n'était jamais entré en vigueur, que la prime de rupture avait été très rarement versée dans les cas analysés, mais surtout que la relation de travail avait été considérablement transformée sous le régime de ce nouveau contrat. Quant à l'accord interprofessionnel puis la loi sur la modernisation du marché du travail, ils ont créé de nouveaux cas de ruptures sans développer de 
véritables sécurités (Freyssinet, 2007 ; Gomel, Méda, Serverin, 2009 ; Freyssinet, 2009).

$\mathrm{Au}$ niveau européen, alors que la flexibilité et la sécurité devaient faire l'objet d'un développement équilibré, les programmes visant à mettre en œuvre la flexibilité ont été bien plus développés. Ils ont fait l'objet d'un suivi, d'une attention et d'une opérationnalisation bien plus intenses que ceux visant à développer la sécurité. Dans les pays où il existait des règles strictes en matière de rupture du contrat de travail ont été développées des mesures visant à faciliter le licenciement ou à recourir à des contrats temporaires. Dans la plupart des pays européens, les règles d'indemnisation du chômage ont été revues, l'offre d'emploi révisée, les dispositifs d'assistance réformés (Serrano 2007; OECD, 2000 et 2007). Partout aussi les programmes d'activation qui pouvaient au départ comporter des dispositions favorables à la formation ont fini par voir considérablement réduite la place accordée à celle-ci par rapport aux emplois aidés ou aux dispositifs visant à pousser les demandeurs d'emploi ou les bénéficiaires de minima sociaux vers l'emploi ; au sein des dépenses de formation, la place accordée aux programmes visant des formations longues (Georges, Grivel, Méda, 2007) a subi le même sort. Par ailleurs, le rapport conjoint du Conseil et de la Commission sur le programme de travail « Éducation et formation 2010 » avait fait état des faibles avancées de l'Europe s'agissant des résultats attendus en matière d'éducation et de formation tout au long de la vie. En réalité, aucun des objectifs fixés n'avait été atteint, qu'il s'agisse des sorties précoces du système éducatif, du nombre de personnes sortant du système éducatif sans qualification ou de l'accès des adultes à la formation en cours de vie active; cet accès restant marqué, entre les pays et entre les différentes catégories de travailleurs, par de fortes disparités. « $L a$ mise en ouvre demeure la principale pierre d'achoppement des stratégies d'éducation et de formation tout au long de la vie » indiquait le $3^{\mathrm{e}}$ rapport conjoint. Le rapport de la Commission européenne sur la mise en œuvre des programmes de life long learning (2008) montrait enfin que $9,6 \%$ des travailleurs de 25 à 64 ans seulement avaient participé, dans les quatre semaines précédentes, à un programme de formation et que celle-ci continuait à bénéficier aux personnes les plus diplômées.
Troisième point en faveur d'un abandon ou du moins d'une certaine méfiance vis-à-vis du concept de flexicurité : le caractère largement contradictoire de la configuration originelle. Avant même la crise, le concept présentait des incohérences qui le rendaient peu « soutenable». Du moins était-il difficile d'imaginer comment autant de contradictions pouvaient être conciliées ou dépassées au sein du concept. Signalons-en deux. La première concerne la coexistence de la « job security » et de l' " employment security ». La job security figure parmi les quatre grands types de sécurité (Wilthagen and Tros, 2004. Elle est censée être faible puisqu'une forte sécurité de l'emploi va de pair avec une forte protection de l'emploi, nuisible puisqu'elle augmente la rigidité du marché du travail. La flexicurité sera donc d'autant plus développée et les performances meilleures que la rupture du contrat de travail sera facile et que la sécurité de l'emploi sera faible. Or, comme l'a parfaitement mis en évidence la contribution de la Fondation de Dublin au débat sur la flexicurité (2008), les éléments nécessaires à une «bonne flexicurité » (notamment les circonstances nécessaires à l'entretien de l'employabilité et au développement d'organisations du travail favorables à l'apprentissage) se développent principalement au cœur du poste de travail et exigent une certaine stabilité de celui-ci. Le développement de l'employabilité est lié à une certaine ancienneté dans l'emploi (autour de 14 ans indique la Fondation) et donc à une certaine sécurité de l'emploi. Les emplois qui présentent un haut niveau d'insécurité objective sont donc les moins susceptibles de développer l'employabilité. Ni l'insécurité de l'emploi, ni le changement d'emploi trop fréquent, ni le passage par le chômage ou les emplois temporaires ne sont favorables à ce qui apparaît, selon la Commission européenne, comme le cœur de la sécurisation des parcours : le développement de l'employabilité.

La sécurité de l'emploi (employment) et la sécurité du revenu (pouvoir bénéficier d'un bon revenu de remplacement) constituent, selon Wilthagen, les deux autres grands types de sécurité. Mais la crainte que le niveau des revenus de remplacement et la durée de perception de ceux-ci soient désincitatifs au retour à l'emploi entraînent une tendance à la diminution et au raccourcissement du versement de ces allocations, de même qu'une préférence donnée aux mesures de 
mise en emploi plutôt que de formation. Les politiques d'activation actuelles apparaissent ainsi en totale contradiction avec une conception large des politiques actives visant à mettre à profit la transition entre deux emplois pour prendre le temps d'améliorer l'employabilité future du travailleur. Le recours plus intense à l'activation, des dépenses mais aussi des personnes, s'accompagne d'une moindre mobilisation des politiques actives du marché du travail, comme l'a d'ailleurs reconnu et revendiqué la Commission (Medeiros, 2006). Les équilibres proposés paraissent donc extrêmement précaires - sauf cas exceptionnel comme le Danemark où l'activation a été longtemps compensée par un haut développement de politiques actives accordant une large place à la formation $(0,5 \%$ du PIB est consacrée à la formation des personnes connaissant un problème sur le marché du travail). Dans ce pays, l'équilibre vient aussi de l'adjonction d'autres politiques.

Enfin, la crise a révélé le caractère insuffisant et non soutenable des fondements de la flexicurité, et plus largement du paradigme général auquel elle se rattache, promouvant l'absence de lien entre le salarié et l'entreprise, l'absence de responsabilisation de l'entreprise dans le devenir de ses salariés, la "légèreté » de la protection sociale... Le concept de flexicurité avait privilégié la mobilité plutôt que la stabilité, l'employabilité et la sécurité prenant appui dans les transitions plutôt que dans le poste ou l'emploi, la facilité à se détacher d'un emploi donné, la préférence accordée au retour très rapide à l'emploi plutôt qu'à l'acquisition de nouvelles compétences à travers des formations ou des requalifications longues. Au moins dans ses usages sinon dans son concept, la flexicurité a privilégié l'absence d'attachement, l'instabilité et la rupture facile du lien sur la stabilité. Or, les pays qui ont le mieux résisté à la crise sont ceux qui au contraire avaient fortement développé leur protection sociale et instauré des liens puissants et difficiles à dénouer entre entreprise et salariés. Tout se passe comme si les pays qui avaient le mieux résisté à la crise et obligé en quelque sorte les entreprises à garder la plus grande partie de leur main-d'œuvre (ce que l'on a appelé la rétention de main-d'œuvre) étaient ceux qui avaient le moins cédé aux sirènes de la déliaison.

Comme de nombreux observateurs l'ont relevé, toutes les économies des pays européens n'ont pas été touchées de la même façon par la crise Les économies les plus exportatrices (Allemagne, Finlande, Hongrie, Danemark, Suède) ou abritant des secteurs très exposés (Espagne pour la construction et Royaume-Uni pour le secteur financier) l'ont été plus que d'autres qui, comme la France, la Belgique ou l'Autriche, dépendent moins des exportations et ont bénéficié de stabilisateurs sociaux venant soutenir la demande (Husson, 2010). Mais c'est également en matière d'emploi que les chocs ont été d'ampleur différente (OCDE, 2010 ; Husson, 2010 ; Erhel, 2010). Si l'Allemagne a subi un choc important sur son PIB (- 6,4\%), son taux de chômage n'a pas varié, alors qu'il a progressé de 2,7 points au Danemark et, pour un choc moindre, de 8,7 points en Espagne. Peut-on proposer l'hypothèse que les pays qui ont le plus développé la flexicurité - c'est-à-dire qui ont réformé leurs marchés du travail en diminuant la protection de l'emploi, en incitant au travail à temps partiel, en préférant l'emploi temporaire à toute autre formule, en promouvant en fin de compte le lien le moins solide possible entre les salariés et les entreprises, tout en prônant des allocations de chômage moins longues et la substitution du work first à la requalification et aux formations longues - sont aussi ceux qui ont enregistré la plus forte réaction de l'emploi à la crise ? Une telle conclusion serait logique, l'effet étant conforme aux objectifs recherchés. N'est-ce pas d'ailleurs ce que l'OCDE reconnaît dans ses Perspectives de l'emploi 2009 en citant des auteurs pour lesquels « il n'existe aucune raison solide de penser que les réformes structurelles récentes ont rendu les marchés du travail de la zone euro beaucoup moins vulnérables aux récessions économiques majeures qu'ils ne l'étaient par le passé » (p. 41). Une autre manière d'écrire cette phrase extraordinairement alambiquée n'est-elle pas de dire que tout incite à penser que les réformes structurelles ont rendu les marchés du travail plus vulnérables ? Mais cette absence de tout lien fort, cette liquidité totale, cette promotion de l'absence de lien n'étaient-elles pas l'objectif recherché ?

Les effets diversifiés de la crise sur l'emploi ont ainsi révélé au grand jour le caractère non soutenable de certains modes de développement, comme celui de l'Espagne, fondé sur une croissance appuyée trop fortement sur une main-d'œuvre peu qualifiée et un poids trop prégnant de l'emploi précaire. Comme 
l'écrit Catherine Vincent, «les performances $d u$ marché du travail espagnol ont été favorisées par des réformes structurelles, d'abord législatives puis contractuelles qui visaient à en accroître la flexibilité. Les réformes ont poursuivi trois objectifs classiques pour faciliter les entrées et sorties sur le marché du travail : assouplir les règles de recours et de renouvellement des contrats temporaires ; favoriser la mobilité géographique et professionnelle; diminuer le coût du licenciement $\gg$. La crise a révélé le caractère insoutenable et court termiste de solutions promouvant la facilitation du licenciement et des politiques d'activation axées sur le work first. En effet, ce genre de politiques a peut-être des effets de court terme « positifs » (amener à l'emploi les personnes en les incitant de toutes les manières et en offrant des morceaux d'emploi) ; cependant, elle n'est pas capable d'accompagner ni une montée en qualification de l'ensemble de la population, ni la mise en place de liens stables entre les entreprises et les salariés en incitant les premières à préférer l'amélioration régulière des compétences de leurs salariés que leur rejet immédiat sur le marché du travail chargé de les former ou de les ramener vers l'emploi.

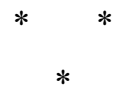

Malgré la crise et, d'une certaine manière, au-delà de la crise, la Commission européenne a conservé à la flexicurité son statut de stratégie prioritaire. La nécessité d'une réforme structurelle du marché du travail semble toujours aussi forte. Le dossier que l'on vient de lire ne fait que renforcer - à la suite d'autres livraisons (on pense à celle de la Revue de l'Ires consacrée à la question, 2009) - les doutes profonds qui sont désormais partagés par une très grande partie de la communauté scientifique sur le caractère adapté de cette stratégie à la gravité des circonstances. Faut-il conserver ce terme et, comme la Confédération européenne des syndicats et le Conseil économique et social européen en 2007, continuer d'espérer que de nouvelles sécurités viendront rééquilibrer un concept que les usages ont fortement infléchi vers la flexibilité ? Ou faut-il plutôt reconnaître que ces usages l'ont désormais rendu impraticable et que la philosophie originelle dans laquelle il est ancré l'empêche à tout jamais d'être le mot d'ordre qui convient? Parmi les différents arguments évoqués dans la dernière partie du présent texte, le fait que la crise ait mis en évidence les piètres performances non seulement des marchés du travail déstructurés par «les liens faibles », mais également des pays qui ont joué le rôle de «modèle », est déterminant. Comme l'illustre le texte de Verd et López-Andreu, la stratégie de flexicurité, telle qu'elle a été conçue, est incapable - en conjoncture «normale » et en cas de crise - de contrebalancer les effets concomitants (ou pire induits par la flexicurité elle-même) d'accentuation de la précarité et de les corriger. C'est aussi pour ces raisons que la plus grande partie des communications présentées lors du colloque Alternatives to Flexicurity: New Concepts and Approaches, co-organisé par the ETUI (European Trade Union Institut), the University of Amsterdam (AIAS/HSI) et the Industrial Relations School (UCM) les 6 et 7 mai dernier à Madrid par Amparo Serrano et Marten Keune, s'orientait vers l'abandon d'un tel concept en faveur d'autres « alternatives » et d'autres «stratégies» fondées sur la qualité de l'emploi, un moment portée par la Commission européenne, ou le travail décent, porté par l'Organisation internationale du travail.

Que pourrait être le cœur de cette alternative? Une grande partie des contributions de ce colloque s'orientait vers une politique du travail décent, ancrée dans une politique des capacités. Accepter un tel tournant impliquerait de renoncer à la priorité accordée par l'Europe à la seule performance économique, pour y substituer une priorité plus large donnée au développement humain, à la cohésion et à la santé sociale et à la possibilité offerte aux citoyens de développer toutes leurs potentialités. C'est cette même volonté, déjà inspirée par Sen, qui avait amené le Programme des Nations Unies pour le Développement (PNUD) à renverser les liens traditionnels entre croissance et développement (voir la série des Rapports sur le développement humain) et à proposer de compléter l'indicateur central de référence, le PIB, par un indicateur plus large : l'IDH (Indice de développement humain) Nous avons aujourd'hui plus que jamais besoin d'une réforme de cette ampleur, capable de recomposer une hiérarchie des priorités, de faire prévaloir la Déclaration de Philadelphie ${ }^{4}(c f$. aussi Supiot (2010),

${ }^{4}$ Cette Déclaration, adoptée en 1944 par la Conférence internatio-
nale du travail, indique que le travail n'est pas une marchandise, 
sur la priorité donnée au commerce et aux marchés financiers, le développement humain sur l'efficacité économique, la préservation du patrimoine naturel et de la santé sociale - condition sine qua non du caractère encore habitable du monde - sur la maximisation du PIB et du chiffre d'affaires. Comme l'indiquent Sen et les auteurs du présent dossier, un tel tournant,

que la liberté d'expression et d'association est une condition indispensable d'un progrès continu, que la pauvreté, où qu'elle existe, constitue un danger pour la prospérité de tous, et que tous les êtres humains, quels que soient leur race, leur croyance ou leur sexe, ont le droit de poursuivre leur progrès matériel et leur développement spirituel dans la liberté et la dignité, dans la sécurité économique et avec des chances égales. qui passe par l'adoption de nouveaux objectifs et de nouveaux indicateurs, est la condition pour que d'autres politiques publiques et d'autres politiques d'entreprises se mettent en place, et d'une manière générale pour que l'ensemble des dispositifs dont l'efficacité n'est évaluée qu'à l'aide de critères très réduits trouve enfin les conditions de leur effectivité.

\section{Mots clés}

Sécurisation des parcours professionnels, approche par les capabilités, Europe

Journal of Economic Literature : J 62, J 68

\section{Bibliographie}

Barbier J.-C. (2007a), « Au-delà de la 'flex-sécurité', une cohérence sociétale solidaire au Danemark», in Paugam S., Repenser la solidarité, l'apport des sciences sociales, Paris, PUF, coll. « Le lien social », pp. 473-490.

Bonvin J.-M., Vielle P. (2009), «Une flexicurité au service des citoyens européens », Revue de l'IRES $\mathrm{n}^{\circ} 63$.

Commission européenne (2007), « Vers des principes communs de flexicurité », (COM (2007)359 final), 27 juin.

Davoine L., Erhel C., Guergoat-Larivière M. (2008), "Monitoring quality in work: European Employment Strategy indicators and beyond", International Labour Review, n 147 (2-3), pp. 163-198.

De Munck et Zimmermann (dir.) (2008), « La liberté au prisme des capacités », Raisons pratiques, Éditions de l'EHESS.

Desrosières A. (2008), Pour une sociologie historique de la quantification, Écoles des Mines, 2008.

Elbaum M. (2006), «La notion d'activation: une pluralité de sens peu utile pour l'action », in Dang A.,
Outin J.-L et Zajdela H. (dir.), Travailler pour être intégré, Mutations des relations emploi-protection sociale, Éditions CNRS, 2006.

Erhel C. (2010), «Les politiques de l'emploi en Europe : le modèle de l'activation et de la flexicurité face à la crise », Documents de travail du CEE 10002, Université Panthéon-Sorbonne (Paris 1), Centre d'Économie de la Sorbonne.

European Foundation for the improvement of Living and Working Conditions (2008), Employment security and employability : a contribution to the flexicurity debate.

European Foundation for the improvement of Living and Working Conditions (2008), More and better jobs.

Ferreras I. (2007), Critique politique $d u$ travail, Presses de Science Po.

Freyssinet J. (2007), «L'accord du 11 janvier 2008 sur la modernisation du marché du travail : un avenir incertain », revue de l'IRES, $\mathrm{n}^{\circ} 54$.

Freyssinet J. (2009), « Ruptures du contrat de travail : les stratégies des acteurs sociaux », in Gomel, Méda, 
Serverin (dir.), L'emploi en rupture, Dalloz, coll. «Études et commentaires ».

Gagel S. (2008), "Expenditure in labour market policies 2005", Statistics focus, n 45.

Georges N., Grivel N., Méda D. (2007), « Les prestations et services d'accompagnement des demandeurs d'emploi : comparaisons internationales », Document de travail du CEE $n^{\circ} 41$.

Gomel B., Méda D., Serverin E. (2009), L'Emploi en rupture, Dalloz.

Gomel B., Méda D., Schmidt N., Dalmasso R. (2007), Le contrat nouvelle embauche, rapport de recherches du Centre d'études de l'emploi.

Husson M. (2009), « Le choc de la crise. Le poids du chômage », Chroniques internationales de l'Ires.

Ires (2009), «Flexicurité, sécurisation des trajectoires professionnelles et protection sociale », Revue de l'IRES, $\mathrm{n}^{\circ} 63$.

Keune M. and Jepsen M. (2007), "Not balanced and hardly new : the European commission's quest for flexicurity”, WP 2007.01, ETUI.

Keune M. and Pochet P. (2009), «Flexicurité en Europe, une approche critique» in Revue de l'Ires, $\mathrm{n}^{\circ} 63$.

Malinvaud E. (2003)», Réformes structurelles du marché du travail et politiques macroéconomiques ", Revue de l'OFCE 3/2003, nº 86, pp. 7-30.

Méda D. (2009), «Flexicurité : quel équilibre entre flexibilité et sécurité ?», Droit social, juin.

Méda D., Minault B. (2005), La sécurisation des trajectoires professionnelles, Document d'études de la DARES.

Medeiros J. (2006), "Effective European Active Labour Market Policies", Speaking note "employment in Europe 2006", November.
OEDC (1994), L'étude de l'OCDE sur l'emploi, Faits, analyses, stratégies.

Tangian A. (2006), "European flexicurity: concepts (operational definitions), methodology monitoring instruments), and policies (consistent implementations)", WSI-Diskussionspapier $\mathrm{n}^{\circ} 148$, October.

Salais R. (2004), « La politique des indicateurs. Du taux de chômage au taux d'emploi dans la stratégie européenne pour l'emploi », in Zimmermann B., Dir., Les sciences sociales à l'épreuve de l'action. Le savant, le politique et l'Europe, Paris, Éditions de la MSH, pp. 287-331.

Serrano Pascual A. (2007) "Reshaping Welfare states:Activation Regimes in Europe" in Serrano Pascual A. and Magnuson L., Reshaping Welfare states and activation regimes, Brussels: Peter Lang.

Serrano Pascual (2008), «Batailles d'idées dans l'espace européen : la lutte contre le chômage et le combat pour le nommer », Revue de l'IRES, $n^{\circ} 60$.

Serrano Pascual A. (2009), «Bataille d'idées dans l'espace européen : la lutte contre le chômage et le combat pour le nommer », la revue de l'IRES, $\mathrm{n}^{\circ} 60$.

Supiot A. (2010), L'esprit de Philadelphie : La justice sociale face au marché total, Éditions du Seuil.

Valeyre A. et Lorenz E. (2005), «Les formes d'organisation du travail dans les pays de l'Union européenne », Travail et Emploi, ${ }^{\circ}$ 102, pp. 91-105.

Vielle P. (2007), Flexicurité : Redéfinir la sécurité des citoyens européens Policy Paper, OSE.

Vincent C. (2009), « Espagne. Le modèle de dialogue social fragilisé par la crise », Chronique internationale de l'Ires, $\mathrm{n}^{\circ} 121$, novembre.

Wilthagen T. and Tros F. (2004), "The concept of flexicurity: a new approach to regulating employment and labour markets", Transfer, vol. 10, $\mathrm{n}^{\circ} 2$, pp. 166-186. 